\title{
Sub Carrier Analysis for QAM Modulation
}

\author{
S. Shanmugasundaram \\ Department of Marine Engineering, AMET University, Chennai, India \\ Corresponding author, e-mail: eei@journal.uad.ac.id
}

\begin{abstract}
Orthogonal Frequency Division Multiplexing (OFDM) based wireless data transmission system is a multi-carrier system in which single higher rate data stream can be divided into multiple lower rate data streams. Modulation and De-Modulation technique play a major role in OFDM based data transmission system. Based on Modulation technique only, the frequency transformation method and encoding and decoding methods are enabled. Effective modulation techniques called as "Quadrature Amplitude Modulation (QAM)" modulation are used to design an OFDM System. Carrier signal is one of the important signals used to modulate the original signal. Analyzing the sub-carrier signal for getting the quality of the modulated signal.
\end{abstract}

Keywords: Quadrature Amplitude Modulation (QAM), sub-carrier signal

\section{Introduction}

OFDM is the modulation as well as multiplexing technique in which single data stream is modulated into multiple data streams each have a lower rate. Hence, an efficient modulation method is required to modulate the digital inputs. For instance, Quadrature Amplitude Modulation (QAM) and Quadrature Phase Shift Keying (QPSK) modulation are the best modulation techniques among different types of modulation techniques [1]. In 4G LTE based mobile broadband communication system, the Quad-QPSK modulation method is used to increase the data rate of transmission [2]. The architecture of FFT has been improvised along with MIMO (Multi-in Multi-out) in OFDM and improves the performances of wireless networks regarding speed [3]. In addition to these performances, orthogonality is the most important property of OFDM signals. Due to orthogonality, the data must be transferred from source to destination without altering any random noise [4]. Interpolation and Decimation are the important property of orthogonality, which helps to reconstruct the original information signal as it is. To re-code, the data from any other format to source format source decoder is used in receiver side of OFDM system. Similarly, source encoder is used on the transmitter side of OFDM system to convert the source information signal into digital input. In another hand, a channel encoder and channel decoder are utilized in the transmitter and receiver part of OFDM system respectively for detecting and correcting a single error or detecting double or triple adjacent errors.

Modulation technique of OFDM signals allows generating the sub-carrier signal from original information signal [5]. Each sub-carrier signal has similar or significantly lower rate data stream. Based on generating a number of sub-carrier signals, total numbers of FFT points are considered. In FFT based frequency transformation technique also, a modulation method is used along with FFT. Different types of digital modulation techniques are available for partitioning single higher rate data stream into some lower rate data streams [6]. Among those more important techniques are Quadrature Amplitude Modulation (QAM), Quadrature Phase Shift Keying (QPSK) modulation and Binary Phase Shift Keying (BPSK) modulation. The modulation method of different input combinations is based on frequency transformation techniques [7]. Binary Phase Shift Keying (BPSK) modulation is one of the necessary modulation techniques among different modulation techniques. The developed CSI based AM method will be useful in upgrading OFDM technologies [8]. 


\section{Sub-Carrier Analysis for QAM Modulation}

Digital amplitude modulation is an alternative principle to digital phase modulation. In digital phase modulation, the amplitude of signals kept no changes and varied the phase of the signals in every step. But, in case of Quadrature Amplitude Modulation (QAM) [9], both amplitude and phase of the signals vary based on input bits. In QPSK modulation, two bits (debits) are to be considered to perform modulation. But, in case of QAM modulation four bits are to be considered to perform modulation. Like QPSK modulation, QAM modulation also has in-phase and Quadrature channel. The advantage of using QAM is that able to carry more bits of information per symbol. The basic block diagram of the QAM modulation is shown in Figure 1. The constellation diagram is shown in Figure 2.

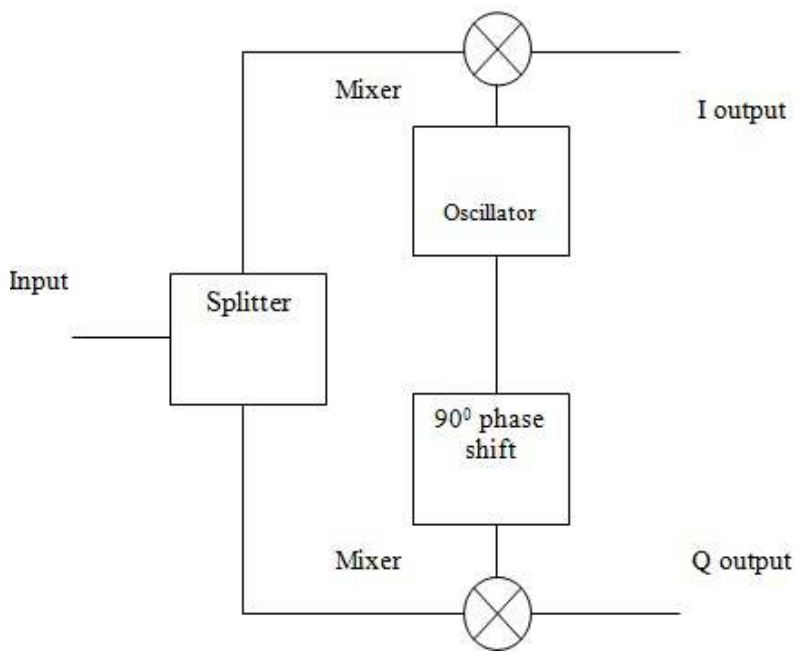

Figure 1. Block diagram of QAM

Cyclic Prefix (CP) and Remove Cyclic Prefix (RCP) are used to reduce the distortion and noise involved in intermediate digital signals. IFFT technique is used to convert the frequency domain signal into time domain signal, and FFT method is used to convert the time domain signal into frequency domain signal. Every block of OFDM transmitter has crucial logics to transfer the data from input to channel. Each sub-carrier signals coming from modulation techniques are orthogonal to each other. The channel of OFDM signals is modelled with Additive White Gaussian Noise (AWGN). These AWGN noises are removed by RCP block.

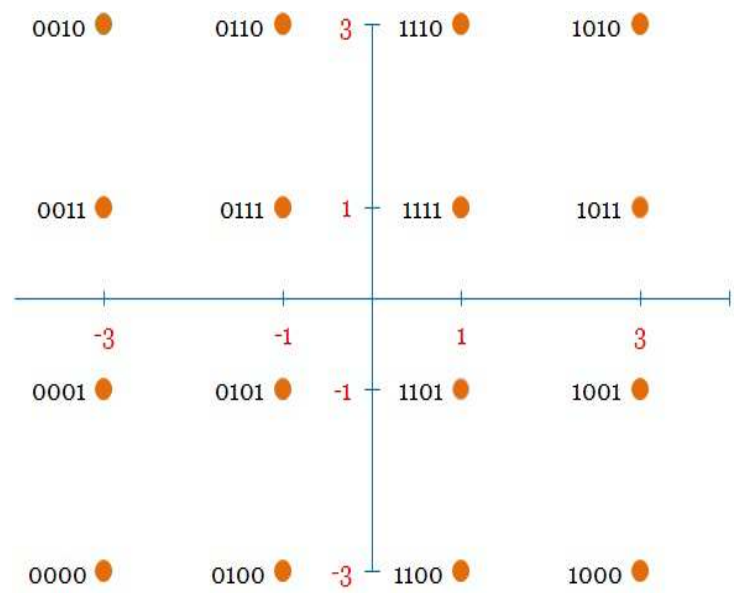

Figure 2. Constellation diagram of QAM 


\section{MATLAB Results for Sub-Carrier}

To analyze the sub-carrier signal and the modulated signal for QAM modulation is evaluated using Matlab tool. The simulation results of original input data shown in Figure 3 and the modulated data using QAM modulation is illustrated in Figure 4. Finally, sub-carrier signals are shown in Figure 5.

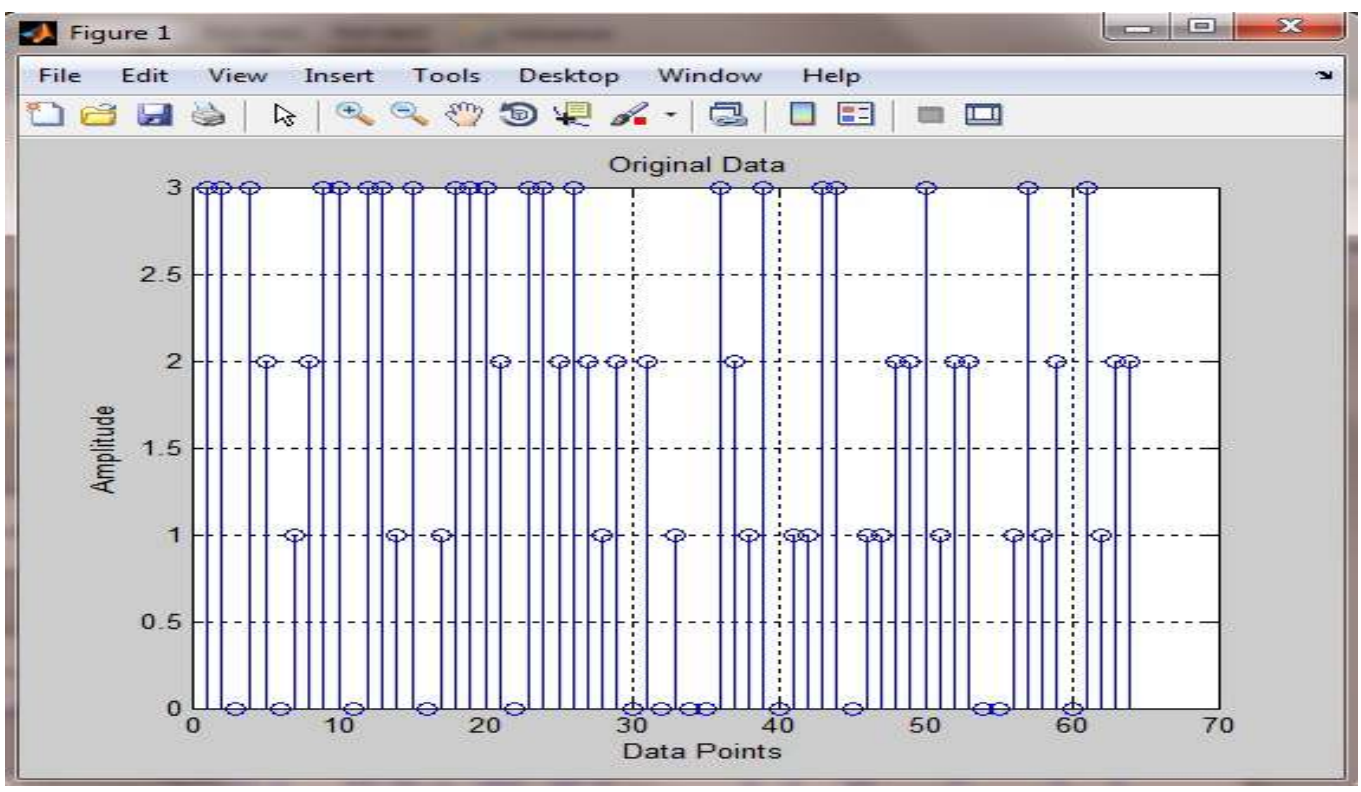

Figure 3. Original data

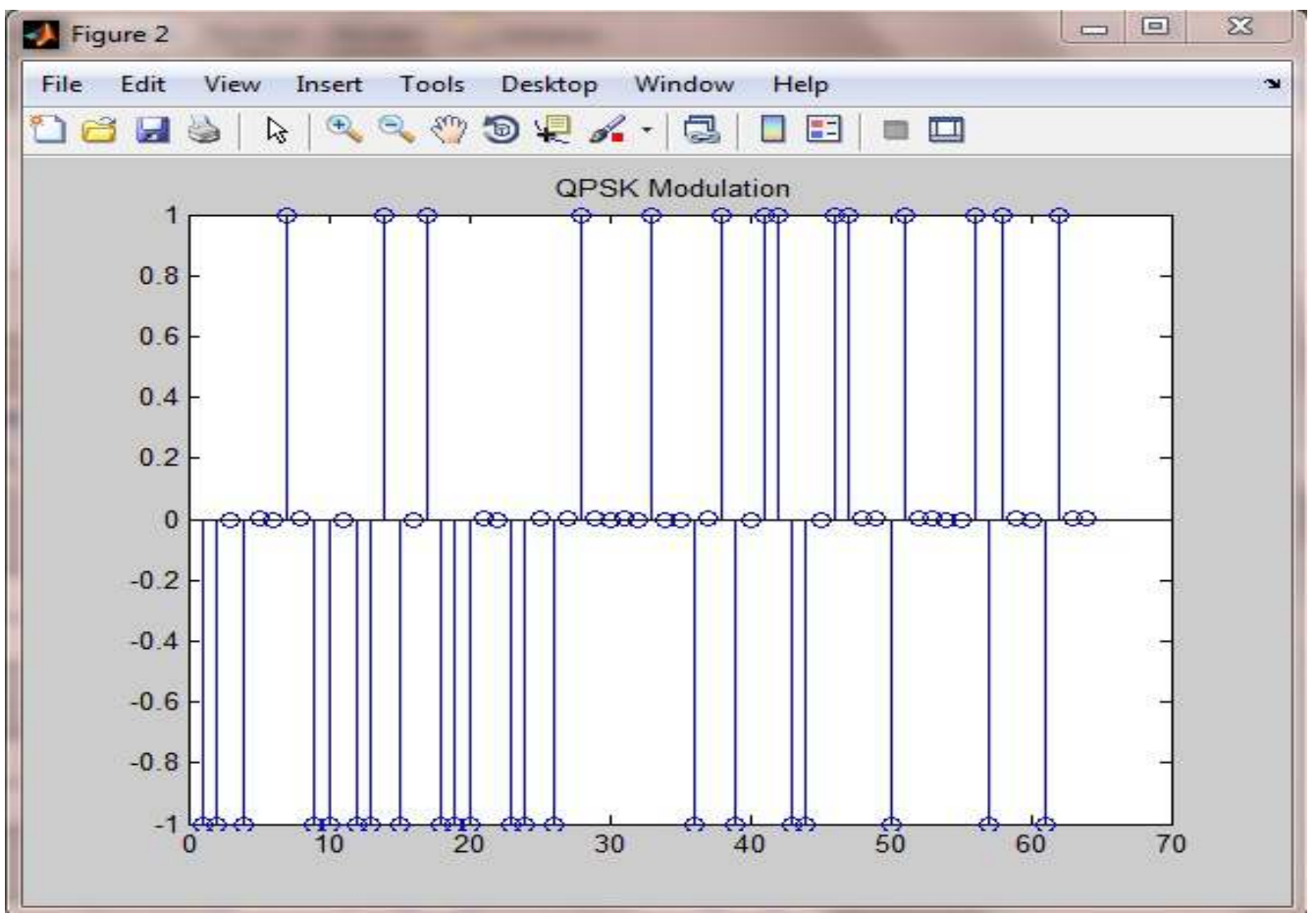

Figure 4. Modulated data 


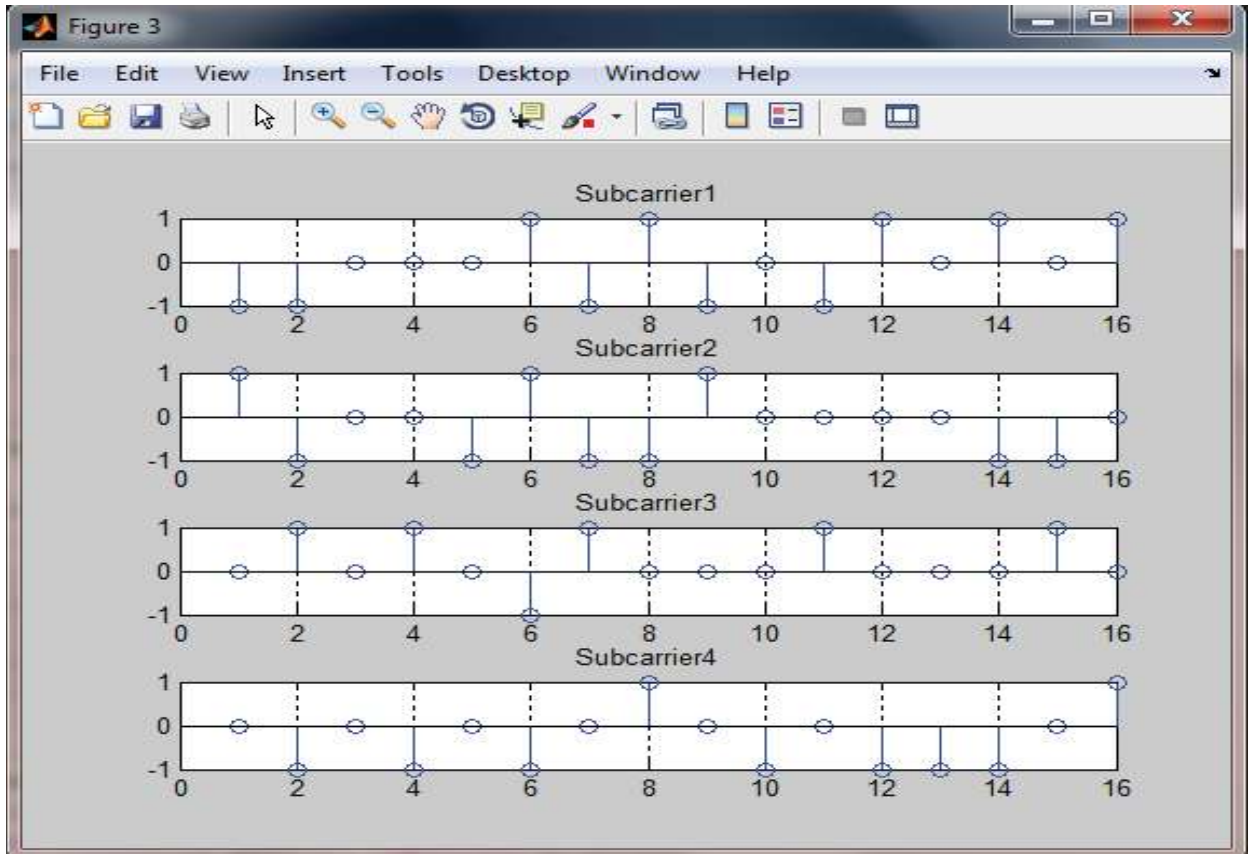

\section{Conclusion}

Figure 5. Sub-Carrier Signal

In this paper, an efficient modulation and demodulation techniques such as Quadrature Amplitude Modulation (QAM) was designed for active data transmission. QAM modulation offers $1.51 \%$ improvements in speed improvements than QPSK modulation. Based on the carrier signal only the modulation acts and delivers the output response. So select the correct and suitable sub-carrier signal for efficient modulation scheme. The designed modulation is suitable for OFDM communication system.

\section{References}

[1] Shatrughna Prasad Yadav, Subhash Chandra Bera. PAPR Reduction for Improved Efficiency of OFDM Modulation for Next Generation Communication Systems. International Journal of Electrical and Computer Engineering (IJECE). October 2016; 6(5): 2310-2321.

[2] Li P, Hamouda W. Performance of Multiple-input and Multiple Output Orthogonal Frequency and Code Division Multiplexing Systems in Fading Channels. IET Communications. 2011; 5(1): 1-11.

[3] Bhagwan Das, Mohammad Faiz Liew Abdullah, Nor Shahida Mohd Shah. A New All-Optical Signal Regeneration Technique for $10 \mathrm{~GB} / \mathrm{S}$ DPSK Transmission System. International Journal of Electrical and Computer Engineering (IJECE). April 2016; 6(2): 859-869.

[4] Sivakumar Reddy B, Lakshmi B. Adaptive Modulation and Coding with Channel State Information in OFDM for WiMax. International Journal of Image, Graphics and Signal Processing. 2015; (1): 61-69.

[5] Agarwal A, Mehta SN. Design and Performance Analysis of MIMO-OFDM System Using Different Antenna Configurations. In Electrical, Electronics, and Optimization Techniques (ICEEOT), International Conference on IEEE. 2016: 1373-1377.

[6] Omar Najah, Kamaruzzaman Seman, Khairi Abdulrahim. Packet Loss Rate Differentiation in slotted Optical Packet Switching OCDM/WDM. Telecommunication Computing Electronics and Control (TELKOMNIKA). 2017; 15(3): 1061-1071.

[7] B U Rindhe, Jyothi Digge, S K Narayankhedkar. Implementation of Optical OFDM Based System for Optical Networks. International Journal of Electrical and Computer Engineering (IJECE). October 2014; 4(5): 767-781.

[8] Haldar D, Panwar S, Kumar V, Goswami A, Dhawan S. Circuits for Optical Based Line of Sight Voice Communication. Bulletin of Electrical Engineering and Informatics (BEEI). 2017; 6(1): 76-80.

[9] Nagaraj V, TR Sumithira, S Prabu. Development of Communication Technologies and Networks for Smart Grid. IJMSR. 2016. 